\title{
GA-BASED TUNING OF NONLINEAR OBSERVERS FOR SENSORELSS CONTROL OF IPMSMs
}

\author{
B. S. Bhangu, C. M. Bingham \\ Department of Electronic and Electrical Engineering \\ University of Sheffield, Mappin Street, Sheffield, S1 3JD, UK \\ Tel. +44 (0) 1142225849 ; Fax. +44 (0) 1142225196 \\ Corresponding author: elp99bsbesheffield.ac.uk
}

Keywords: Sensorless Estimation techniques, Interior Permanent Magnet Motors.

\begin{abstract}
The paper considers two observer-based rotor position estimation schemes for sensorless control of Interior Permanent Magnet Synchronous Machines (IPMSMs). Emphasis is given to techniques based on feedbacklinearisation followed by Luenberger observer design, and direct design of non-linear observers. Genetic Algorithms (GAs) based on the principles of evolution, natural selection and genetic mutation are employed to address difficulties in selecting correction gains for the observers, since no analytical tuning mechanisms yet exist, with results included to demonstrate the enhanced performance attributes offered by observers tuned in this way.
\end{abstract}

1

\section{Introduction}

The power-to-weight advantages and thermal characteristics attributed to permanent magnet (PM) brushless motors are making them a preferred option for electro-mechanical actuation and servo equiptment for future vehicle systems. However, intense economic constraints, product volumes and manufacture/motor parameter tolerance issues consistent with the requirements of vehicle technology industries, for instance, has meant that the cost and component count attributed to the requirement for rotor position sensors for the increasing number of machines envisaged for auxiliary systems, is leading to greater interest in sensorless position estimation schemes.

For servo-type applications, and those where electromagnetic torque ripple and audible noise have a significant impact on overall product quality, vector control schemes are preferred. In these cases, sensorless operation is achieved by utilising only motor terminal quantities to accurately estimate rotor position. The situation is further complicated for cost-critical applications by the favoured use of IPMSMs that possess significant saliency, thereby presenting a significant challenge for the designer in the field. Whilst various observer-based techniques have been proposed for non-salient PMSMs
$[1,2,3,4,5,6]$ an integrated approach to their design for application to salient machines remains outstanding.

A methodology developed solely in the $d-q$ reference frame that employs an inner-loop non-linear feedback linearization controller to render the system as essentially linear for the subsequent design of an observer using classical Luenberger techniques [3], is initially considered. Subsequently, a full non-linear observer scheme developed in the $\alpha$ - $\beta$ stator-fixed reference frame, is presented. The structural difference between the techniques is shown in Fig. 1.

A performance comparison between various observer techniques for use with non-salient PMSMs, using an Extended Kalman Filter (EKF) as a benchmark, has been previously reported in $[7,8]$, where the advantages of integrating load torque estimates is also considered. However, these techniques are now extended specifically for application to salient machine counterparts, with the use of GAs to assist with selection of optimal observer gains.

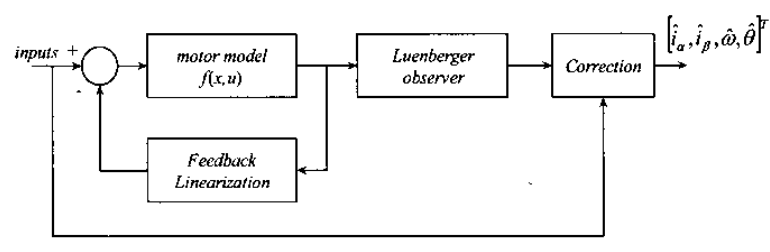

(a) Feedback linearization and Luenberger observer.

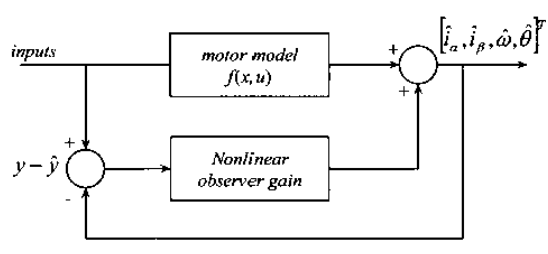

(b) Nonlinear observer.

Fig. 1 Structure of observer schemes 


\section{Impact of Saliency on Rotor Position Estimates}

The use of flat buried magnets is often preferred in the design of custom motors for cost-critical applications since they can provide similar air-gap flux densities to surface mounted counterparts whilst being cheaper to manufacture and require less bulk material. A consequence, however, is that the direct and quadrature inductances, $L_{d}$ and $L_{q}$, respectively, may not be equal, differing often by up to $30 \%$. Employing observers on buried magnet machines without accommodating the effects of saliency can be significantly detrimental to the accuracy of resulting rotor position estimates [7]: Extending the techniques to accommodate saliency is therefore necessary.

\subsection{Feedback Linearization and Luenberger Observer Design Incorporating the Effects of Saliency}

A dynamic model of an IPMSM can be described in the $d-q$ reference frame by (1), where the applied inputs are $u=\left[v_{d}, v_{q}\right]^{T}$, and the measured outputs are the machine terminal currents $y=\left[i_{d}, i_{q}\right]^{T}$.

$$
\begin{aligned}
& \frac{d}{d t} i_{d}=-\frac{R_{s}}{L_{d}} i_{d}+\omega i_{q} \frac{L_{q}}{L_{d}}+\frac{v_{d}}{L_{d}} \\
& \frac{d}{d t} i_{q}=-\frac{R_{s}}{L_{q}} i_{q}-\omega i_{d} \frac{L_{d}}{L_{q}}-\frac{K_{e}}{L_{q}} \omega+\frac{v_{q}}{L_{q}} \\
& \frac{d}{d t} \omega=\frac{K_{t} p}{J} i_{q}+\frac{3 p}{2 J}\left(L_{d}-L_{q}\right) i_{d} i_{q}-\frac{B}{J} \omega \\
& \frac{d}{d t} \theta=\omega
\end{aligned}
$$

$R_{s}, L_{d}, L_{q}, K_{e}$ and $K_{t}$ are, respectively, the phase resistance, $d$ axis and $q$-axis inductance and back-emf and torque constants. $J$ is the rotor inertia, $B$ is the motor viscous friction, $\omega$ is the rotor angular velocity, $\theta$ is the rotor position and $p$ denotes the number of pole-pairs.

From (1), it is evident that equations describing the electrical dynamics are coupled by nonlinear angular velocity terms. Such nonlinearities can be accommodated by introducing auxiliary inputs (2) that apply nonlinear feedback of applied voltages [3] and angular velocity (estimated in practice).

$$
\begin{aligned}
& u_{d}=v_{d}+\omega i_{q} L_{q} \\
& u_{q}=v_{q}-\omega i_{d} L_{d}
\end{aligned}
$$

resulting in the new dynamic description:

$$
\begin{aligned}
\frac{d}{d t} i_{d} & =-\frac{R_{s}}{L_{d}} i_{d}+\frac{u_{d}}{L_{d}} \\
\frac{d}{d t} i_{q} & =-\frac{R_{s}}{L_{q}} i_{q}-\frac{K_{e}}{L_{q}} \omega+\frac{u_{q}}{L_{q}} \\
\frac{d}{d t} \omega & =\frac{K_{t} p}{J} i_{q}+\frac{3 p}{2 J}\left(L_{d}-L_{q}\right) i_{d} i_{q}-\frac{B}{J} \omega \\
\frac{d}{d t} \theta & =\omega
\end{aligned}
$$

This procedure constitutes traditional feedback linearization for a non-salient PMSM. However, due to effects of saliency, a non-linear term remains in the model (see $\dot{\omega}$ ) by virtue of the non-zero term $\left(L_{d}-L_{q}\right)$, thereby preventing classical Luenberger-type observer techniques to be subsequently applied.

A solution to this problem is to introduce an additional state variable, $z$, as follows,

$$
z=i_{d} i_{q}
$$

with the resulting time-derivative being,

$$
\frac{d}{d t} z=-R_{s}\left(\frac{1}{L_{d}}+\frac{1}{L_{q}}\right) z+\frac{U_{z 1}}{L_{d}}+\frac{U_{z 2}}{L_{q}}
$$

where,

$$
\begin{aligned}
& U_{z 1}=u_{d} i_{q} \\
& U_{z 2}=-K_{e} \omega i_{d}+u_{q} i_{d}
\end{aligned}
$$

resulting in:

$$
\frac{d}{d t}\left[\begin{array}{c}
i_{d} \\
i_{q} \\
\omega \\
z
\end{array}\right]=\left[\begin{array}{c}
-\frac{R_{s}}{L_{d}} i_{d}+\frac{u_{d}}{L_{d}} \\
-\frac{R_{s}}{L_{q}} i_{q}-\frac{K_{e}}{L_{q}} \omega+\frac{u_{q}}{L_{q}} \\
\frac{K, p}{J} i_{q}+\frac{3 p}{2 J}\left(L_{d}-L_{q}\right) z-\frac{B}{J} \omega \\
-R_{s}\left(\frac{1}{L_{d}}+\frac{1}{L_{q}}\right) z+\frac{U_{z 1}}{L_{d}}+\frac{U_{z 2}}{L_{q}}
\end{array}\right]
$$

In view of the fact that terms $U_{21}$ and $U_{z 2}$ in (6) are directly dependent on the auxiliary inputs, they can be considered as known disturbances, for convenience. Hence, a state variable representation of the machine (6), suitable for the design of a Luenberger observer, can be derived (7): 


$$
\begin{aligned}
\frac{d}{d t}\left[\begin{array}{l}
i_{d} \\
i_{q} \\
\omega \\
z
\end{array}\right] & {\left[\begin{array}{cccc}
-\frac{R_{s}}{L_{d}} & 0 & 0 & 0 \\
0 & -\frac{R_{s}}{L_{q}} & -\frac{K_{e}}{L_{q}} & 0 \\
0 & \frac{K_{t} p}{J} & -\frac{B}{J} & -\frac{3 p}{2 J}\left(L_{d}-L_{q}\right) \\
0 & 0 & 0 & -R_{s}\left(\frac{1}{L_{d}}+\frac{1}{L_{q}}\right)
\end{array}\right]\left[\begin{array}{l}
i_{d} \\
i_{q} \\
\omega \\
z
\end{array}\right] } \\
& +\left[\begin{array}{cc}
\frac{1}{L_{d}} & 0 \\
0 & \frac{1}{L_{q}} \\
0 & 0 \\
0 & 0
\end{array}\right]\left[\begin{array}{l}
u_{d} \\
u_{q}
\end{array}\right]+\left[\begin{array}{cc}
0 & 0 \\
0 & 0 \\
0 & 0 \\
\frac{1}{L_{d}} & \frac{1}{L_{q}}
\end{array}\right]\left[\begin{array}{l}
U_{z 1} \\
U_{z 2}
\end{array}\right]
\end{aligned}
$$

The structure of the resulting observer is given by:

$$
\dot{\hat{x}}=A \hat{x}+B u+w+K(y-\hat{y})
$$

where $w$ is the 'known' disturbance. Notably, the state variable relating to $\theta$ is omitted in (6-8) since it is wellknown that the model formulation in $d-q$ co-ordinates renders $\theta$ to be unobservable after feedback linearization (for the non-salient case). Convergence dynamics for $\theta$ is then ensured using a correction term proposed in [9]. The complete rotor position estimation scheme therefore consists of i) a feedback linearising controller, ii) a linear observer \& iii) a correction scheme to allow $\hat{\theta}$ to converge to $\theta$, as shown in Fig. 2.

Design of the observer gain matrix, $K$, is motor dependent, but consists of assigning eigenvalues of the observer to be sufficiently fast compared to the motor electrical dynamics so that the transient convergence of the state estimates has minimal impact on the outer-loop control system.

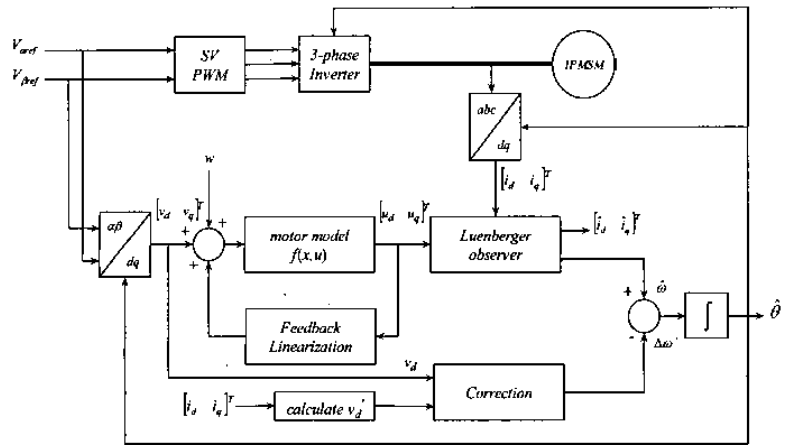

Fig. 2. Luenberger observer employed for rotor position estimation of IPMSM.

The validity of the proposed observer scheme is demonstrated on a candidate $42 \mathrm{~V}$ IPMSM with $28 \%$ saliency. Results neglecting the effects of saliency in the observer, Fig. 3, show a resulting steady state error in angular velocity and a corresponding divergence of rotor position estimates, for a step change in speed demand. However, whilst increasing observer complexity, incorporating effects of saliency into the observer allows stability to be retained, see Fig. 4.

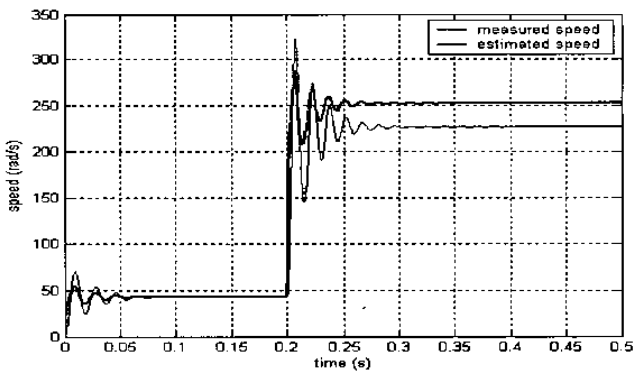

(a) Actual and estimated rotor speed.

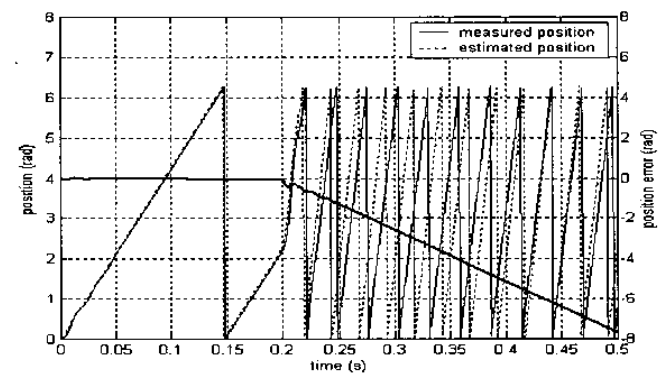

(b) Actual and estimated rotor position.

Fig. 3. Results from a salient machine without incorporating saliency.

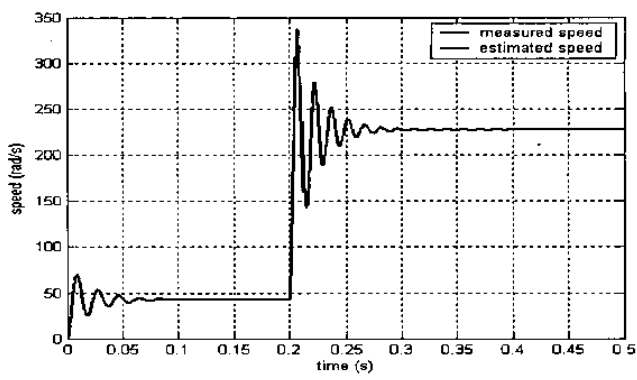

(a) Actual and estimated rotor speed.

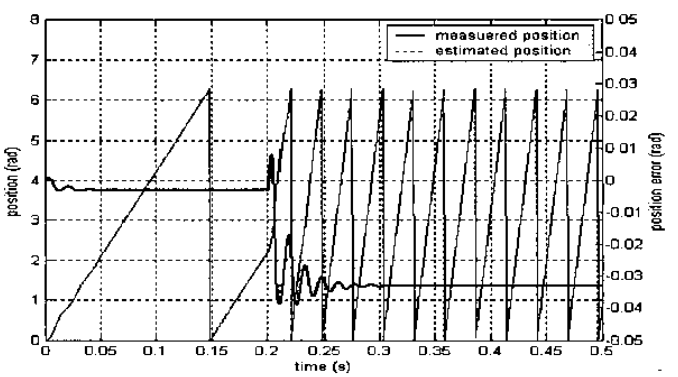

(b) Actual and estimated rotor position and position error.

Fig. 4. Results with saliency incorporated into the observer. 
2.2 Non-linear observer scheme incorporating the effects of saliency

A full account of the formulation of the proposed direct nonlinear observer is beyond the scope of this paper. However, the reader is referred to [7] for an expanded treatment. The underlying structure is ultimately given by:

$$
\begin{aligned}
& \frac{d}{d t}\left[\begin{array}{c}
\hat{i}_{\alpha} \\
\hat{i}_{\beta} \\
\hat{\omega} \\
\hat{\theta}
\end{array}\right]=\left[\begin{array}{c}
-\frac{R_{s}}{L_{d}} i_{\alpha}+\frac{K_{e}}{L_{d}} \omega \sin \theta-\omega L_{o} i_{q} \cos \theta-i_{q} L_{o} \sin \theta+\frac{v_{\alpha}}{L_{d}} \\
-\frac{R_{s}}{L_{d}} i_{\beta}-\frac{K_{e}}{L_{d}} \omega \cos \theta-\omega L_{o} i_{q} \sin \theta+i_{q} L_{o} \cos \theta+\frac{v_{\beta}}{L_{d}} \\
\frac{K_{t} p}{J}\left(\hat{i}_{\beta} \cos \hat{\theta}-\hat{i}_{\alpha} \sin \hat{\theta}\right)+\frac{3 p}{2 J}\left(L_{d}-L_{q}\right)_{d} i_{q}-\frac{B}{J} \hat{\omega} \\
\hat{\omega}
\end{array}\right] \\
& +K(y-\hat{y})
\end{aligned}
$$

where, the observer gain $K$ takes the form,

$$
\begin{array}{ccc}
K=\left[\begin{array}{c}
K_{1} \\
\Gamma_{s} K_{2}
\end{array}\right] & K_{1}=K_{v}\left[\begin{array}{ll}
\mathrm{I} & 0 \\
0 & \mathrm{I}
\end{array}\right] \\
\Gamma_{s}=\frac{L_{d}}{K_{e} \omega}\left[\begin{array}{cc}
\omega \sin \theta & -\omega \cos \theta \\
\cos \theta & \sin \theta
\end{array}\right] & K_{2}=K_{2}\left[\begin{array}{ll}
\mathrm{I} & 0 \\
0 & \mathrm{I}
\end{array}\right] \\
L_{0}=\frac{L_{d}-L_{q}}{L_{d}} &
\end{array}
$$

It is noted that no formal tuning methods have been reported for selecting $K_{y}$ and $K_{z}$, and recourse to either 'sweepparameter' methods or intelligent tuning methods is therefore still required.

\section{Tuning the Nonlinear Observer}

\subsection{Parameter Sweep}

Here, sweep tests are performed to identify stable regions associated with successful sensorless operation. By way of example, Fig 5(a) shows a boundary between stable and unstable regions of observer estimates, with the stable region represented by results of 0 and the unstable region represented by results of \pm 1 . Further, within the stable region of gains, Fig. 5(b) shows position error obtained for a range of values of $K_{y}$ and $K_{z}$, for the correction gain. In this case it can be seen that enhanced performance is obtained with $K_{y}$ bounded between $4000 \rightarrow 6000$ and $K_{z}$ bounded between $0.5 \rightarrow 1 \times 10^{6}$.

\subsection{Genetic Algorithm Implementation}

An alternative technique is to select observer gains based on the results from a GA tuned on-line. A block diagram representation of the basic operation of a GA, with the objective of obtaining minimum position error, is shown in Fig. 6. In principle, 'parents' are selected for reproduction with a bias towards individuals with a 'high-fitness', and reproduced by cross breeding or recombining by processes of crossover and mutation [10] to generate off-spring. Repetition of this process leads to evolutionary populations that enhance the fitness function [10] (position error in this case).

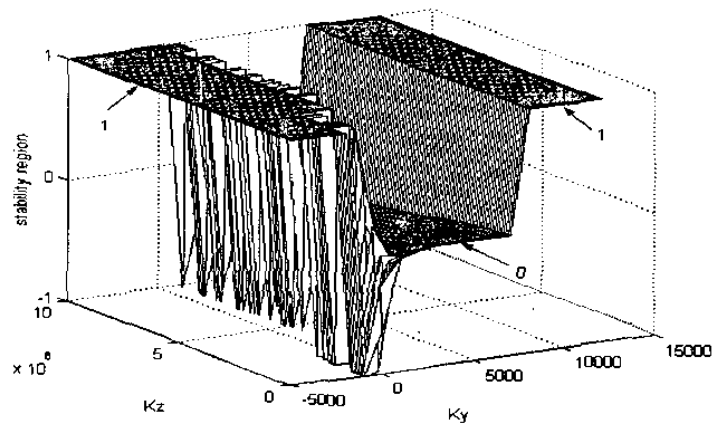

(a)

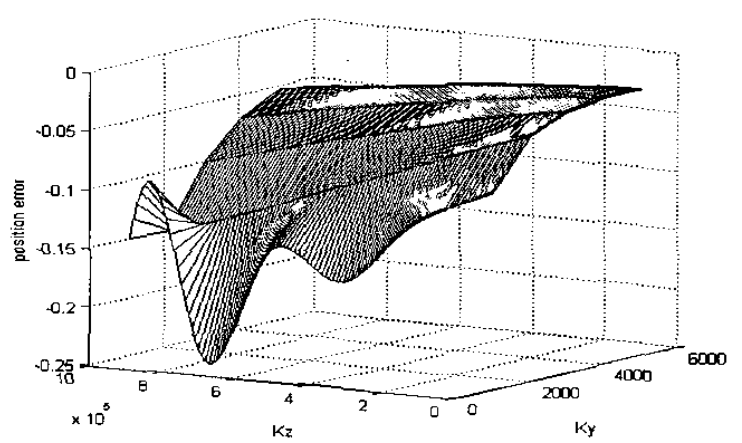

(b)

Fig. 5., Stability and performance for a range of $K_{y}$ and $K_{z}$.

Results from the candidate IPMSM, Fig. 7, show the GA exploring a large state space to maximise the fitness function i.e. minimum rotor position error (Fig. 7(a)). Figure 7(b) shows the rotor position error obtained from initial observer gains (obtained from the 'sweep test' on a trial-and-error basis) and with resultant gains obtained from GA trials. It is seen that the GA selects gains that generate smaller transient overshoot, lower settling time and reduced steady state error. It should be noted that in general a drawback of employing GAs is that several extensive tests are often required to ensure that the fitness function does not become trapped in a local minima, and therefore provide a degree of confidence of obtaining optimal gain solutions.

\section{Conclusions}

Two observer-based rotor position estimation schemes for sensorless control of IPMSMs have been discussed, the first employing a linearised controller and a second scheme considering a complete non-linear implementation. For the latter, difficulties in tuning the observer gain terms have prompted an investigation into the use of parametric 'sweep' tests and GAs, with tuning based on GAs showing a marked performance improvement. 


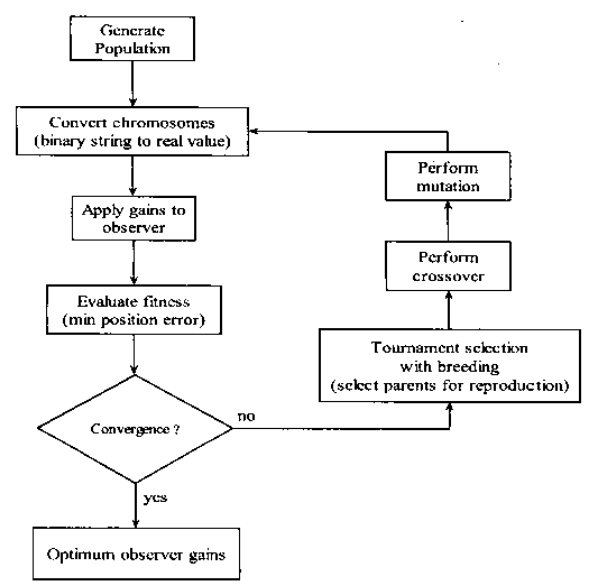

Fig. 6. GA for tuning nonlinear observer.

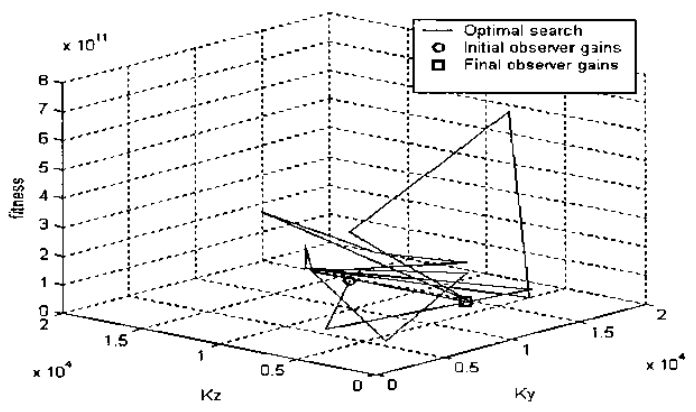

(a) On-line selection of observer gains for optimum fitness.

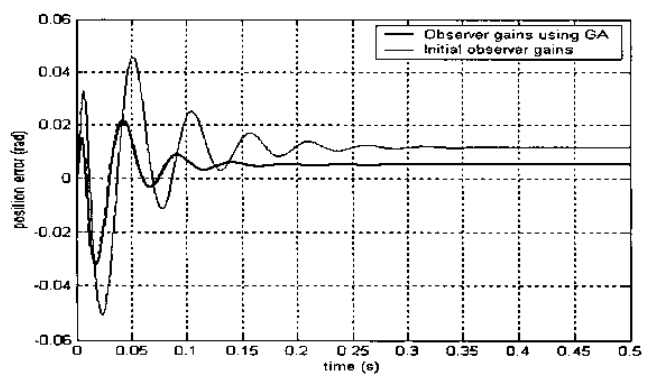

(b) Position error associated with using GA observer gains.

Fig. 7. Simulated results from automotive salient machine.

Importantly, for cost critical applications it is highly desirable that such observer schemes make use of existing processing hardware. In this case, computational overhead and memory requirements are of significant importance. Table $\mathrm{I}$ is therefore included to show the resource requirement for both techniques, with a corresponding EKF implementation (a traditional solution to such problems) being included as a benchmark. In this case, the feedback linearisation/Luenberger observer technique is seen to provide the highest resource efficiency.

\begin{tabular}{lcc}
\hline & Computation time & Memory requirement \\
\hline EKF & 1 & 1 \\
Luenberger & 0.10 & 0.34 \\
Nonlinear & 0.17 & 0.42 \\
\hline
\end{tabular}

Table I. Observer resource requirements

\section{References}

[1] A. Bado, S. Bologani, M. Zigliotto "Effective estimation of Speed and Rotor Position of an PM Synchronous Motor Drive by a Kalman Filtering Technique" IEEE Proc. PESC, 1992, pp.951-957.

[2] G. Zhu, A. Kaddouri, L. Dessiant, O. Akhrif "A Nonlinear State Observer for the Sensorless Control of a Permanent-Magnet AC Machine" IEEE Trans. Ind. Electronics, vol. 48, 2001, pp.1098-1108.

[3] D. Hamada, K. Uchida, L. F, Yusivar, S. Wakeo and T. Onuki "Sensorless Control of PMSM using a Linear Reduced Order Observer including Disturbance Torque Estimation" EPE'99 - Lausanne, Waseda University.

[4] J. Solsona and C. Muravchik "A Nonlinear Reduced Order Observer for Permanent Magnet Synchronous Motors" IEEE Trans. Ind. Electronics, vol. 43, 1996, pp.492-497.

[5] R. Dhaouadi, N. Mohan and L. Norum, "Design and Implementation of an Extended Kalman Filter for the State Estimation of a Permanent Magnet Synchronous Motor" IEEE Trans. on Power Electronics, vol. 6, 1999, pp.491-497.

[6] S. Bolognani, R. Oboe and M. Zigliotto, "Sensorless FullDigital PMSM Drive With EKF Estimation of Speed and Rotor Position" IEEE Trans. Ind. Electronics, vol. 46, 1999, pp.184-191.

[7] B. Bhangu, C. M. Bingham, "Nonlinear State-Observer Techniques for Sensorless Control of Automotive PMSM's, including Load-Torque Estimation and Saliency"; EPE 2003, Toulouse, France.

[8] B. Bhangu, C. Williams, C. M. Bingham and J. Coles "EKFs and other Nonlinear State-Estimation Techniques for Sensorless Control of Automotive PMSMs"; Proc. SPEEDAM 2002, pp.C5 33-38, Italy.

[9] N. Matsui and M. Shigyo, "Brushless de Motor Control without Position and Speed sensors" IEEE Trans. Ind. Electron., vol. 28, 1992, pp. 120-127.

[10] L. Feng, Y. Qiwen, "Applications of GAs in Sensorless Speed adjustment Control for Induction Motor Drive System" Power Electronics and Motion Control Conf., Proc. PIEMC 2000, vol. 1, pp. 296-301. 